\title{
Investigation of the shear-layer instabilities in supersonic impinging jets using dual-time velocity measurements
}

\author{
T. Sikroria ${ }^{1 *}$, J. Soria ${ }^{2}$, S. Karami ${ }^{2}$, R. Sandberg ${ }^{1}$, A. Ooi ${ }^{1}$ \\ ${ }^{1}$ Department of Mechanical Engineering, University of Melbourne, Parkville Campus, Melbourne, \\ Victoria - 3010, Australia. \\ ${ }^{2}$ Laboratory for Turbulence Research in Aerospace and Combustion (LTRAC), Department of Mechanical \\ and Aerospace Engineering, Monash University, Clayton Campus, Melbourne, Victoria - 3800, Australia. \\ *tsikroria@student.unimelb.edu.au
}

\section{Introduction}

Motivated by applications in the propulsion industry, the fundamental study of phase-locked shear-layer instabilities in supersonic impinging jets has been of research interest for long time. While such flows have been experimentally investigated in various research studies using time-unresolved particle image velocimetry (PIV) techniques, the understanding of the shear-layer dynamics is limited, due to the absence of temporal information. Time-resolved PIV measurements for high-speed flows require a large bandwidth, which is challenging to achieve with the current state of technology. An alternate approach using timeunresolved double-PIV measurements is presented in the current study, which provides multiple samples of dual-time data, depicted in figure 1. Such data can be obtained using two co-visual PIV systems, triggered at a user-selectable time-offset, $\Delta t$. As shown by Sikroria et al. (2020), the application of techniques like dynamic mode decomposition (DMD) on time-unresolved dual-time data provides valuable information about the flow structures governing the shear-layer instabilities. The experimental setup for such measurements in supersonic impinging jets, followed by the determination of the relevant dynamical flow structures from the data, will be presented in the conference.

\section{Experimental measurements}

The experiments were conducted using the sub- and super-sonic jet facility in the Laboratory for Turbulence Research in Aerospace and Combustion (LTRAC), Monash University, whose details have been reported in Mitchell et al. (2013), Weightman et al. (2019). The main challenge in double-PIV experimental measurements was to ensure that the camera from the first PIV system did not capture the illumination by the laser from the second PIV system and vice versa. The principle of the difference in polarization between two laser systems was used for the measurements in the current study, which has been used previously in the work of Christensen and Adrian (2002). The optical setup used for the experiments is shown in figure 2. A high-power and high-frequency Innolas SpitLight DPSS EVO IV laser, which was a composite of two Taser systems, the MASTER and the SLAVE, respectively, was used for the double-PIV experiments. The MASTER and the SLAVE lasers had opposite polarization and this difference in the polarization formed the basis of the dual-time data acquisition. The images were captured using two cameras, mounted on a T-shaped plate. Both the cameras were connected to a polarizing beam splitter, attached to the lens. The arrangement ensured that each of the two cameras could see the laser light corresponding to a particular polarization only. The timing was synchronized by programming the BBB (Beagel Bone Black) controller (Fedrizzi and Soria (2015)) which provided the platform to specify a user-selectable time-shift, $\Delta t$, between the two PIV systems. Multi-grid cross-correlation algorithm proposed by Soria (1996) was used for the processing of images.

Following the work of Sikroria et al. (2020), the double-PIV measurements were carried out for moderately under-expanded jet at a nozzle pressure ratio (NPR) of 3.4 and impinging distance of 5 nozzle diameters. Acoustic measurements were additionally conducted for the selected test-case, for validation of 

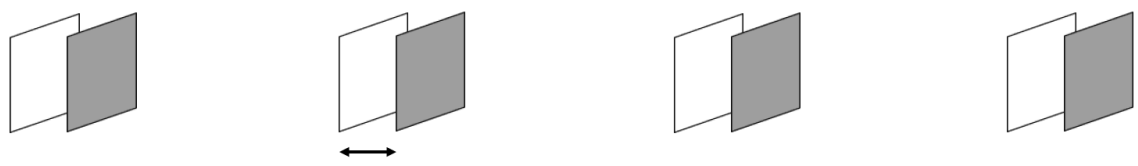

$\Delta \mathrm{t}$

Figure 1: Sampling of velocity fields in double-PIV measurements showing ensemble of pairs of velocity fields.

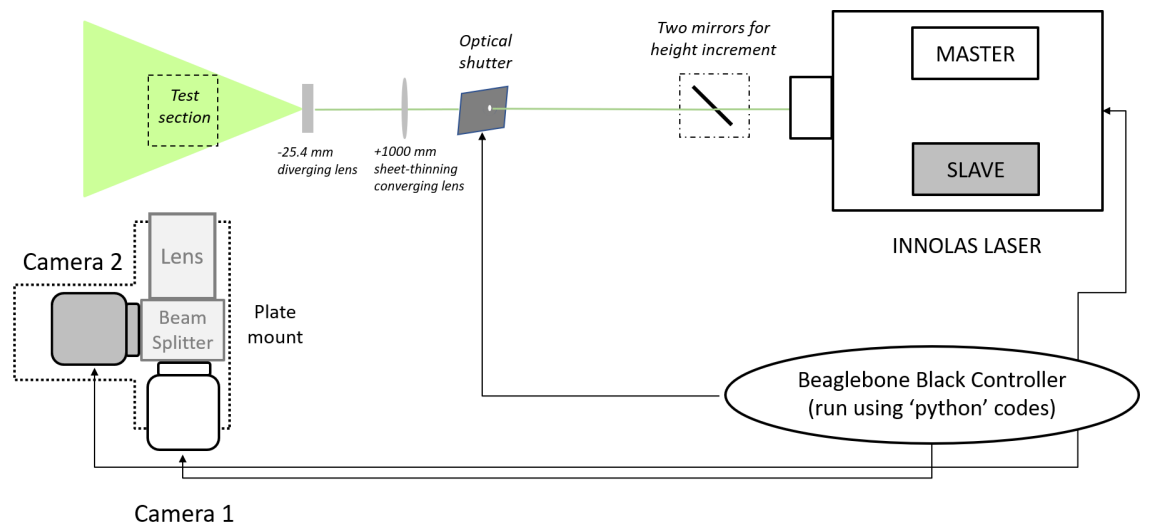

Figure 2: Schematic of the optical setup for double-PIV measurements.

the spectral information obtained from the double-PIV data. G.R.A.S. 46BE 1/4" CCP free-field standard microphone, having a frequency range of $4 \mathrm{~Hz}-80 \mathrm{kHz}$, was used for the acoustic measurements. The impingement tones were determined from the peaks observed in the spectrum obtained using the pressure signals from the microphone.

\section{Acknowledgements}

The research is being funded by a Discovery Project Grant from the Australian Research Council (ARC).

\section{References}

Christensen K and Adrian R (2002) Measurement of instantaneous eulerian acceleration fields by particle image accelerometry: method and accuracy. Experiments in Fluids 33:759-769

Fedrizzi M and Soria J (2015) Application of a single-board computer as a low-cost pulse generator. Measurement Science and Technology 26:095302

Mitchell DM, Honnery DR, and Soria J (2013) Near-field structure of underexpanded elliptic jets. Experiments in fluids 54:1578

Sikroria T, Soria J, Karami S, Sandberg RD, and Ooi A (2020) Measurement and analysis of the shear layer instabilities in supersonic impinging jets. in AIAA AVIATION 2020 FORUM. page 3070

Soria J (1996) An investigation of the near wake of a circular cylinder using a video-based digital crosscorrelation particle image velocimetry technique. Experimental Thermal and Fluid Science 12:221-233

Weightman JL, Amili O, Honnery D, Edgington-Mitchell D, and Soria J (2019) Nozzle external geometry as a boundary condition for the azimuthal mode selection in an impinging underexpanded jet. Journal of Fluid Mechanics 862:421-448 